\title{
The Prototype of a Wideband Ku-Band Conical Corrugated Horn Antenna with 3-D Printing Technology
}

\author{
Mustafa Emre Carkaci ${ }^{1}$, Mustafa Secmen ${ }^{1 *}$ \\ ${ }^{1}$ Department of Electrical and Electronics Engineering, Yasar University, Izmir, Turkey \\ *Corresponding author, E-mail: mustafa.secmen@yasar.edu.tr
}

\begin{abstract}
This study is about the design and production of a conical corrugated horn antenna used to feed reflector antennas in satellite communication (direct broadcast satellite-DBS) systems. The antenna designed with CST Microwave Studio program operates within wideband of $10.5-18.5 \mathrm{GHz}$ at $\mathrm{Ku}-$ band. The prototype is realized with new generation 3D printing technology and conductive paint coating method, which makes the antenna lightweight and provides low cost and faster production. According to measurement results, the antenna has return loss almost better than $20 \mathrm{~dB}$, gain value of minimum $14.5 \mathrm{dBi}$ and sidelobe level of $-18 \mathrm{~dB}$ at most within 1.76:1 frequency bandwidth. Antenna is observed to have a gain loss of at most $1.5-2 \mathrm{~dB}$ within the band as compared to the same antenna with high conductivity metal, which needs higher cost and production time for the manufacturing.
\end{abstract}

\section{Introduction}

The corrugated horn antennas can be designed in different configurations as E-plane, H-plane, pyramidal and conical. However, the conical type of corrugated horns is frequently used in dual or circular polarization applications such as satellite communication or direct broadcast satellite (DBS). These type of horn antennas which have slots and teeth in its inner surface are used as a feed for reflectors in communication satellites, radar and remote sensing systems and also they are used as direct radiator antennas for broadband measurements. The antenna is preferred due to their high directivity and gain as well as low crosspolarization level, low side and back lobe levels and good return loss value. All of these advantageous features result from the fact that inner surface is in a corrugated structure. Corrugated horn antennas basically consist of four main sections, which are input (feed) waveguide, transition, corrugated profile (mode converter) and antenna aperture. When there is $\mathrm{TE}_{11}$ mode propagation in the input waveguide section, which is a smooth and flat walled structure, $\mathrm{TE}_{11}$ and $\mathrm{TM}_{11}$ modes propagates in the corrugated surface. The propagation of the both electric and magnetic field components of TE and TM modes are at the same velocity, which results in as a single hybrid $\left(\mathrm{HE}_{11}\right)$ mode [1]. Therefore, the corrugated surface of the antenna actually behaves like a mode converter. Hybrid mode propagation provides extremely good beam symmetry in radiation patterns with low cross-polarization levels, with high beam efficiency. For these reasons, corrugated horns provide good wide-bandwidth performance.

Since, these antennas are complicated in their geometric shapes, high precision is required in the production process. Nowadays, computer-controlled precision milling $(\mathrm{CNC} / \mathrm{CCM})$ machines are often used for the production of these complex structures. However, it can be stated that $\mathrm{CNC} / \mathrm{CCM}$ process is challenging, long-running and very high-cost from the point of view of a product, which is designed and will be produced as a prototype. Thanks to development of additive manufacturing technology, prototype production has become possible with a much lower cost as well as in a much shorter time by using 3-D printing machines. Although there are other technologies in 3-D printing such as stereolithography (SLA), Polyjet and others, most of 3-D printers on the market work with FDM (Fused Deposition Modeling) technology due to its advantages in terms of cost of the machine and materials used in the production, compact size of the machine, common usage and easy operation in the production process. FDM based 3-D printers can produce a desired structure by melting various plastic materials such as acrylonitrile butadiene styrene (ABS) or polylactic acid (PLA) and extruding layer by layer [2]. Another advantage of FDM technology is that the printing sensitivity settings could be changed by the user's preference. Many parameters, such as layer height, print speed and infill density can be adjusted with the desired precision by user to create better quality prints.

In this study, a prototype of Ku-band corrugated conical horn antenna is verified by using an alternative manufacturing technique with 3-D FDM/PLA printing technology and nickel conductive aerosol painting. Since the production of antennas and electromagnetic structures with 3-D printing technology also becomes a hot topic due to their low cost and low production time, there are many studies in recent years in literature, which presents the verification of horn antennas or waveguide structures at different frequency bands with 3-D printing technology [412]. However, only the studies of [4-7] include an antenna some of which contain narrow bandwidth designs when 20 $\mathrm{dB}$ or even $15 \mathrm{~dB}$ return loss bandwidth is considered [6, 7]. Besides, some of the mentioned studies in literature use 
SLA or Polyjet techniques where FDM is preferred in this study in order to obtain the prototype with as low cost and low production time as possible. The ones using FDM technology produce their structures with ABS material where PLA is selected in this study due to its advantages over ABS in the manufacturing process to be described in the following sections.

The designed conical corrugated horn antenna operates at $\mathrm{Ku}$-band with the measured return loss better than almost $17 \mathrm{~dB}$, gain higher than $14.5 \mathrm{dBi}$ and peak sidelobe level of $-18 \mathrm{~dB}$ within the frequency band of $10.5-18.5 \mathrm{GHz}$.

\section{Design and Simulation Works}

\subsection{Antenna Design Procedure}

The corrugated conical horn antenna is designed to operate within wideband between 10.5 and $18.5 \mathrm{GHz}$ to cover general RX band (10.5-12.75 GHz) and TX band (17.3-18.4 $\mathrm{GHz}$ ) in DBS communication [13], and TX/RX bands $(10.7-12.75 \quad \mathrm{GHz}$ and $13.75-14.5 \mathrm{GHz})$ in telemetry/telecommand satellite applications [14]. The designed antenna structure is depicted in Figure 1, which includes three parts, which are rectangular-to-circular transition, circular waveguide and corrugated section.

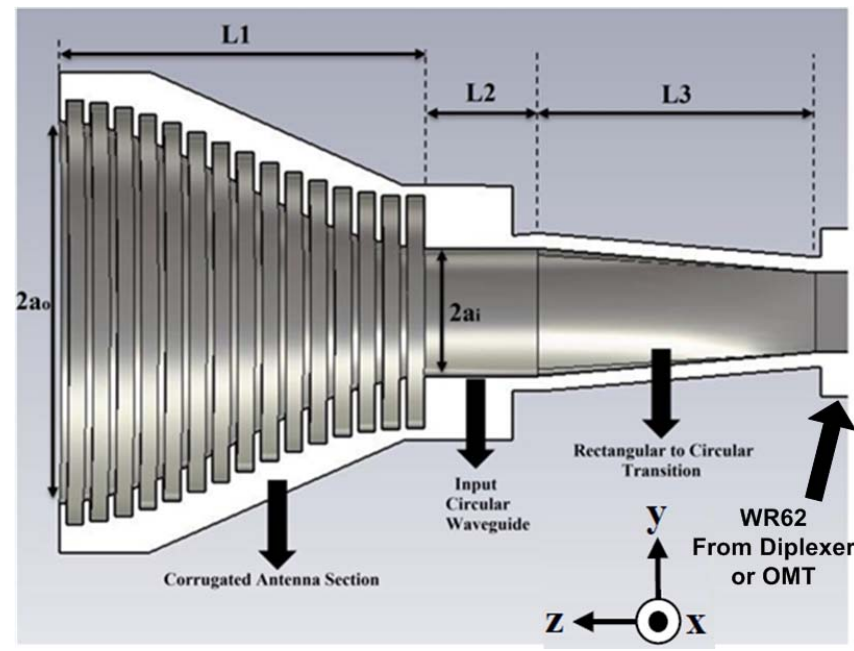

(a)

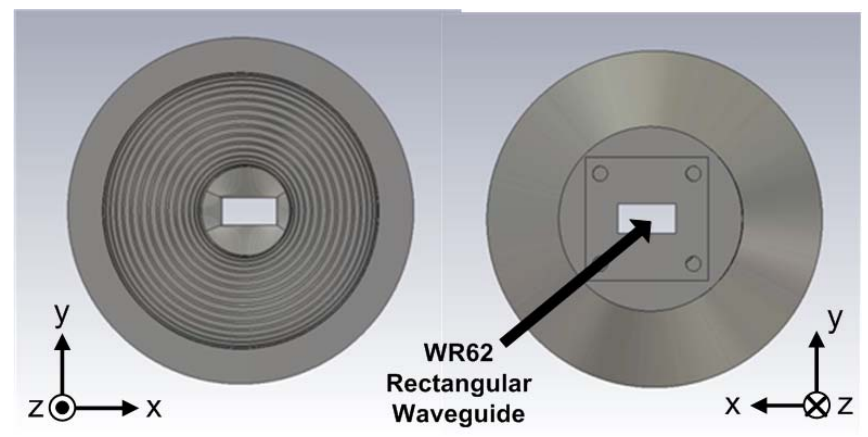

(b)

Figure 1: The views of the designed corrugated conical horn antenna (a) Cross section (side) view (b) Front and back views.
The antenna is fed by a standard rectangular waveguide of WR62 to make the operation bandwidth within 10.5-18.5 $\mathrm{GHz}$ as much as possible. As shown in Figure 1, it can be either fed by a diplexer or an orthomode transducer (OMT) in a reflector antenna feed system. Then, in order to provide matching between input circular waveguide of the corrugated antenna and the feed rectangular WR-62 waveguide with inner dimensions of about $15.8 \mathrm{~mm}$ by 7.9 $\mathrm{mm}$, a circular-to-rectangular transition structure is used. The final parts of the overall antenna in Figure 1 are the input circular waveguide and corrugated profile.

The initial dimensions of corrugated profile (geometry) of the antenna are obtained by using theoretical formulations/design procedures described in literature [3, 15]. The input radius of the antenna (the parameter of $a_{i}$ in Figure 1) is approximately calculated with the expression of

$$
a_{i}=3 \lambda_{0, m} /(2 \pi)
$$

where $\lambda_{0, m}$ is free space wavelength at the frequency equal to $f_{m} \approx 1.2 f_{\min }$. Here, $f_{\min }$ is the minimum frequency in the design, which is $10.5 \mathrm{GHz}$ in the proposed structure. According to design procedures in $[3,15]$, the radius of the aperture (the parameter of $a_{0}$ in Figure 1) should be equal to or greater than $\lambda_{0, m}$ such that $a_{o} \geq \lambda_{0, m}$. In the design procedure, the specification of minimum $15 \mathrm{dBi}$ gain within the frequency band of $10.5-18.5 \mathrm{GHz}$ is handled. Therefore, the initial dimension of aperture radius is calculated to satisfy this specification by also keeping the aperture radius as small as possible to make the antenna compact. Then, the initial dimension for length of corrugated part (the parameter of L1 in Figure 1) is found by evaluating the knowledge that the optimum flare angle for horn antenna should be selected as almost $19^{\circ}-20^{\circ}[4,15]$. In the corrugated antenna designs, corrugation depths should be generally between $\lambda_{\min } / 4$ and $3 \lambda_{\max } / 4$ along the band where $\lambda_{\min }$ and $\lambda_{\max }$ are free space wavelength values for maximum and minimum frequencies in the design, respectively. The initial dimensions for corrugations are also determined by considering the stated range.

The length of the transition structure, the parameter of L3 in Figure 1 , is initially taken as approximately $2 \lambda_{\mathrm{g} \text {,average }}$ at the center frequency $(14.5 \mathrm{GHz})$ to obtain good matching [16] where $\lambda_{\mathrm{g} \text {,average }}$ is the mean value of guided wavelengths for rectangular waveguide of WR62 and circular waveguide with radius of $\mathrm{a}_{\mathrm{i}}$ at the center frequency of $14.5 \mathrm{GHz}$.

After the determination of the initial dimensions, these dimensions are optimized to give final structure dimensions by using the simulations with CST Microwave Studio 2017. In the optimization process, In addition to the desired specification of minimum $15 \mathrm{dBi}$ gain, it is also aimed to have return loss higher than $20 \mathrm{~dB}$ in the optimization process. In the initial simulation works carried out by CST, the material of all parts in the overall antenna is selected as Perfect Electric Conductor (PEC) to speed up the optimization process. Besides, the results with PEC material are used as reference results for comparison such that they can be approximately taken as the results when the antenna is manufactured with $\mathrm{CNC}$ machine and high conductivity material such as aluminum. 
Table 1: Final dimensions of designed antenna.

\begin{tabular}{cc}
\hline Parameter & Size $[\mathrm{mm}]$ \\
\hline ao & 37.88 \\
ai & 12.64 \\
L1 & 72 \\
L2 & 22.5 \\
L3 & 55 \\
\hline
\end{tabular}

As the results of optimization process, the final dimensions depicted in Figure 1 are given in Table 1. The corrugated part has constant corrugation values of $1.4 \mathrm{~mm}$ thickness and $3.4 \mathrm{~mm}$ spacing. The depth values of corrugations are initially arranged to give a linear tapering with about $19.3^{\circ}$ flare angle. The depth values of first and last corrugations (closest and furthest corrugations to input waveguide) are found as $10.35 \mathrm{~mm}$ and $4.68 \mathrm{~mm}$, respectively.

The simulation results (reflection coefficient and gain values) of the designed antenna with the dimensions given in the previous paragraphs and PEC material is shown in Figure 2. According to results demonstrated in Figure, the return loss and gain values are found to be greater than 20 $\mathrm{dB}$ and $15 \mathrm{dBi}$, respectively, for the frequency band 10-19 GHz. As described in Section 1, these results can be also treated as the results of the antenna fabricated with $\mathrm{CNC}$ machine and processed with high conductivity material although no manufacturing is realized with $\mathrm{CNC} /$ high conductivity material in this study.

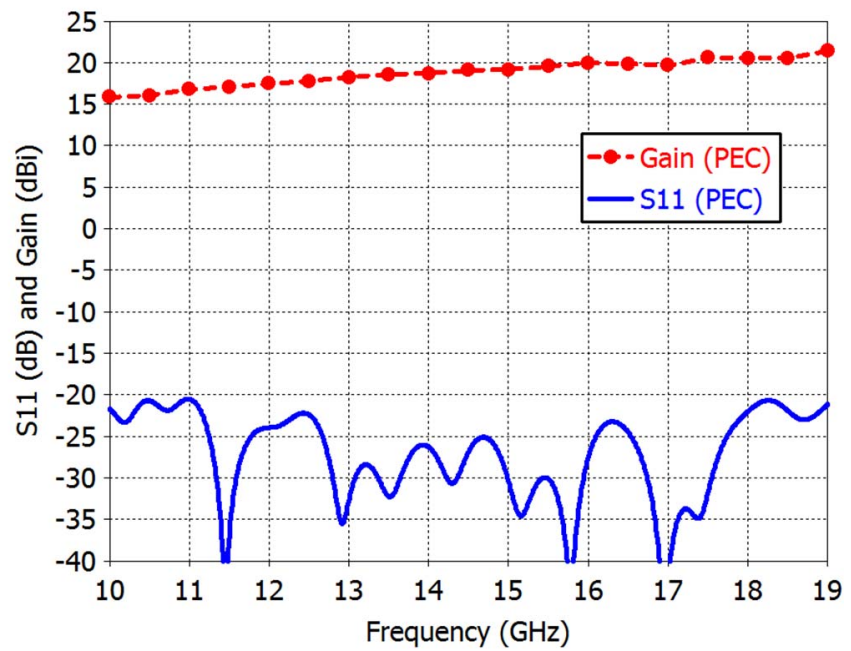

Figure 2: The simulation results of the designed antenna with PEC material for $\mathrm{S}_{11}$ (reflection coefficient) and realized gain.

\subsection{Simulation Works with 3-D Printing and Coating Materials}

After the design procedure and simulations employed with PEC material as mentioned in Section 1, the simulations are applied for the antenna structure to be produced with 3-D printing and coating technique in order to examine the effects of the technique on the antenna performance. For this purpose, since the dielectric material of polylactic (PLA) with a relative permittivity of about $\varepsilon_{\mathrm{r}}=2.8$ and loss tangent of $\tan \delta=0.011$ is to be used in 3-D printing manufacturing steps, PEC material in the simulations in
Section 2.1 is replaced by PLA for the new simulations. For the coating material, a low cost product of Super Shield-841 nickel conductive aerosol paint is used [17]. Same material is applied in the study of [4] where the surface resistance at around $10 \mathrm{GHz}$ was found to be around $R_{s}=2.5-3 \Omega / \square$. Therefore, the surface resistance of the paint material is chosen to be $2.5 \Omega / \square$ at $10 \mathrm{GHz}$ in the new simulations. According to datasheet in [17], the conductivity of the material is given as approximately $\sigma=2.4 \times 10^{4} \mathrm{~S} / \mathrm{m}$. Therefore, by considering the surface resistance of $R_{S}=2.5$ $\Omega / \square$, the skin depth and relative permittivity values at 10 $\mathrm{GHz}$ are evaluated as about $\delta_{\mathrm{s}}=17 \mu \mathrm{m}$ and $\mu_{\mathrm{r}}=4$, respectively, by using the equations given below.

$$
\begin{gathered}
R_{s}=\frac{1}{\sigma \delta_{s}} \\
\delta_{s}=\sqrt{\pi f \mu \sigma}
\end{gathered}
$$

In order to make the thickness of the coating much greater than skin depth, it is selected to be about $0.1 \mathrm{~mm}$, which corresponds to three coats for Super Shield-841 [17], and this thickness value is taken as coating thickness on PLA in the following simulations.

The selected coating material is actually a moderately lossy conductor due to its moderate conductivity. The loss values might be reduced by using silver coated copper conductive paint such as Super Shield-843 with higher conductivity [18] instead of Super Shield-843. However, it is more expensive than Super Shield-841. Therefore, since the main of the study is to demonstrate the validation of the production of a corrugated horn at $\mathrm{Ku}$-band with low cost as much as possible, relatively lossy but cheap coating is used. After the insertion and replacement of proper materials mentioned above, the simulation results of the new structure with 3-D printing and coating materials. The results for reflection coefficients $\left(\mathrm{S}_{11}\right)$ and gain are given in Figure 3 and Figure 4, respectively, along with the ones belonging to the antenna with PEC material for the comparison.

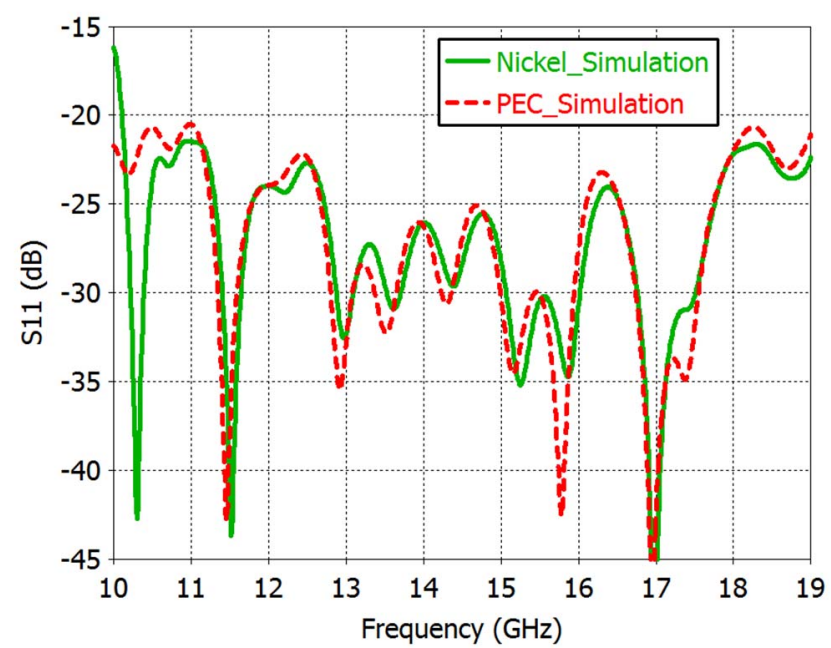

Figure 3: The simulation results for $\mathrm{S}_{11}$ (reflection coefficient) of the proposed antennas with PLA/nickel materials and PEC material. 


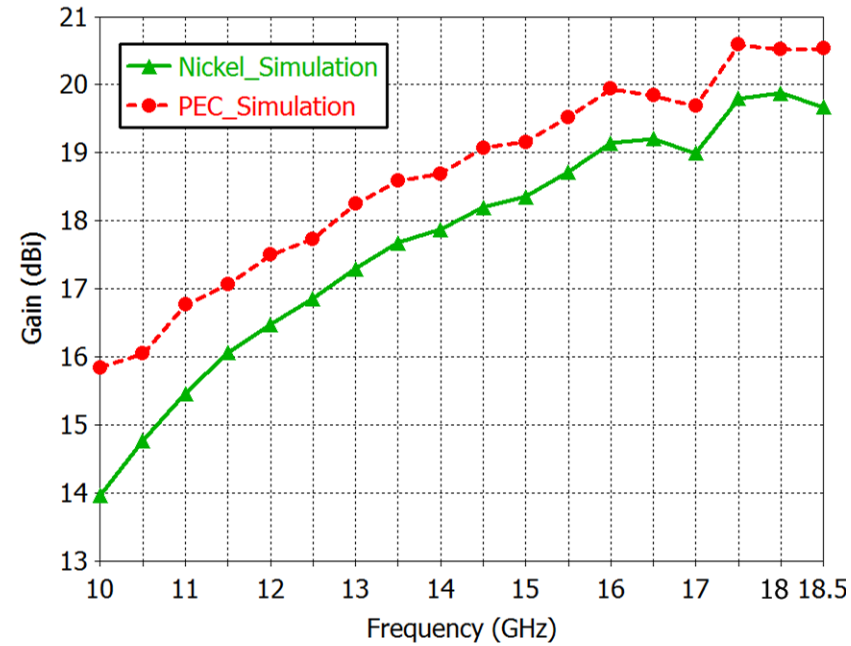

Figure 4: The simulation results for the gain of the proposed antennas with PLA/nickel materials and PEC material.

From the simulation results given for the coated antenna, it can be concluded that PLA/nickel materials have quite slight effect on the return loss performance of the antenna where the return loss values are almost above $20 \mathrm{~dB}$ for 10 $19 \mathrm{GHz}$. The values slightly drop below $20 \mathrm{~dB}$ for the frequencies around $10 \mathrm{GHz}$. This is due to the reason that the coating thickness on PLA decreases the inner dimensions of the antenna including the feed rectangular waveguide. Therefore, the cutoff frequency of the waveguide (and the antenna) becomes closer to $10 \mathrm{GHz}$, at which the return loss of the antenna is deteriorated. When the gain values in Figure 4 are examined where the values for PEC structure can be also considered as simulated directivity values of the antenna, it can be observed that the radiation loss in gain is about $1-1.5 \mathrm{dBi}$ at the given band. The difference in gain values between PEC and nickel coating can increase up to $2 \mathrm{dBi}$ at $10 \mathrm{GHz}$ due to the reason of decrease in the dimension (or increase in the cutoff frequency) mentioned above. When the desired frequency band of $10.5-18.5 \mathrm{GHz}$ in the design is considered, the simulations of the antenna with PLA/nickel provide return loss more than $20 \mathrm{~dB}$ and gain higher than almost $15 \mathrm{dBi}$ within the given band.

After the return loss and gain performances of the antenna with PLA/nickel obtained with simulations, the radiation pattern characteristics are examined. For this purpose, the gain patterns for lowest $(10.5 \mathrm{GHz})$, center $(14.5 \mathrm{GHz})$ and highest $(18.5 \mathrm{GHz})$ frequencies within the given band are selected. The corresponding normalized co-polarization patterns are given in Figure 5 for H-plane (xz plane or $\phi=$ $0^{\circ}$ plane in Figure 1) and E-plane (yz plane or $\phi=90^{\circ}$ plane in Figure 1). From the results given in Figure 5, it can be stated that the sidelobe levels for E-plane is greater than $\mathrm{H}$ plane as expected. The sidelobe levels for E-plane are found as $-21.9 \mathrm{~dB},-31.3 \mathrm{~dB}$ and $-19.0 \mathrm{~dB}$ for $10.5,14.5$ and 18.5 $\mathrm{GHz}$, respectively. By considering H-plane $\left(\phi=0^{\circ}\right.$ cut or azimuth plane) and E-plane $\left(\phi=90^{\circ}\right.$ cut or elevation plane), the maximum sidelobe level within the frequency band of $10.5-18.5 \mathrm{GHz}$ is gathered as $-19 \mathrm{~dB}$, which is quite sufficient for corrugated horn antenna applications.

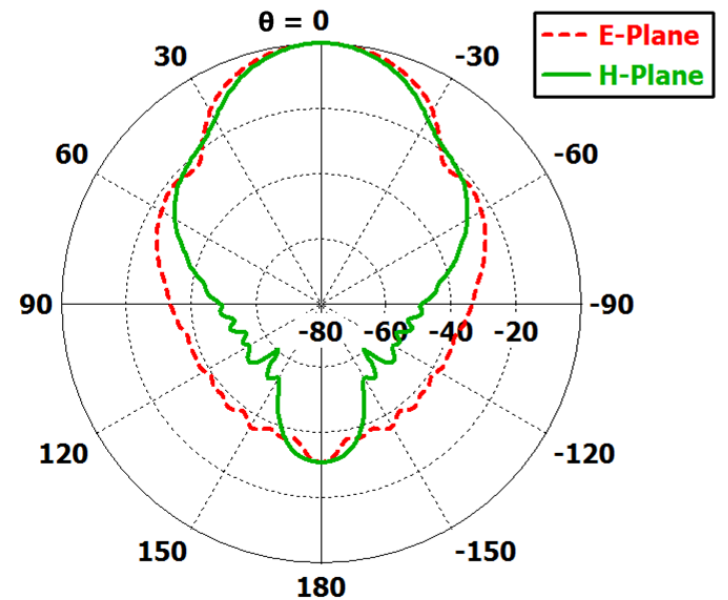

(a)

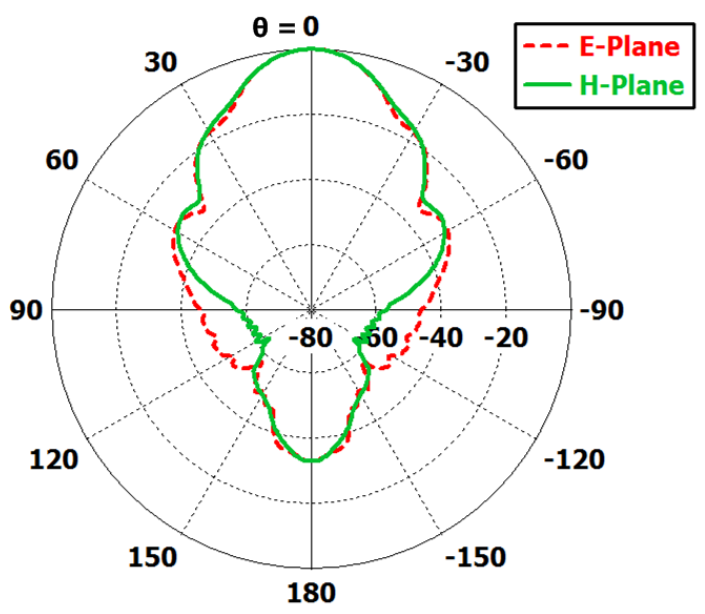

(b)

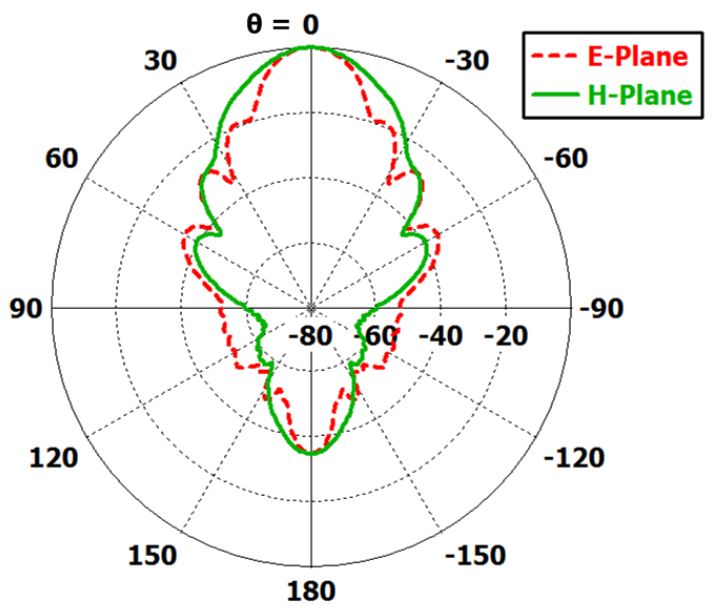

(c)

Figure 5: The simulated co-polarization normalized patterns (E-plane and H-plane) of the designed antenna with PLA/nickel for (a) $10.5 \mathrm{GHz}$ (b) $14.5 \mathrm{GHz}$ (c) $18.5 \mathrm{GHz}$.

The cross polarization performance of the antenna with PLA/nickel is also satisfactory that unnormalized crosspolarization gain values are found be at most $-55 \mathrm{dBi}$ over the full $\mathrm{H}$-plane and E-plane $\left(\phi=0^{\circ}\right.$ and $90^{\circ}$ cuts $)$ within the frequency band. When co-polarization gain values in 
Figure 4 are considered, the cross polarization discrimination (XPD) values are found to be higher than 60$65 \mathrm{~dB}$ at E- and H-planes even for 10-dB beamwidth in the band. For $\phi=45^{\circ}$ cut at which the maximum crosspolarization (or minimum XPD) values are generally observed for conical corrugated horn antennas [19], XPD values are calculated to be better than $20 \mathrm{~dB}$ for $3-\mathrm{dB}$ beamwidth and $10 \mathrm{~dB}$ for $10 \mathrm{~dB}$-beamwidth in the given frequency band.

\section{Manufacturing Steps}

For the fabrication of the antenna structure, a 3-D printer (Ultimaker 2+) is used, and PLA filament is selected as printing material. The important reason to choose this material is due to fact that PLA is easier to be printed than ABS. This is because high temperature is required to print ABS material and 3-D printers require a closed area to maintain the heat. Another reason for the choice of PLA is being cheaper than ABS and environmentally friendly [2]. The printed halves of the manufactured antenna, which are identical parts, are as shown in Figure 6(a). Consequently, they could be easily coated and made conductive after the printing is completed. For the printing process, $0.4 \mathrm{~mm}$ diameter nozzle is used by applying $0.2 \mathrm{~mm}$ layer height, $\% 50$ infill density and $50 \mathrm{~mm} / \mathrm{s}$ print speed precision settings.

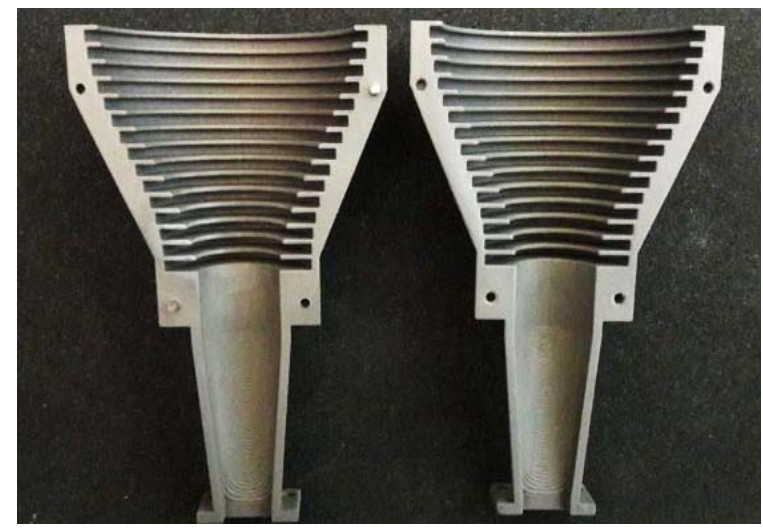

(a)

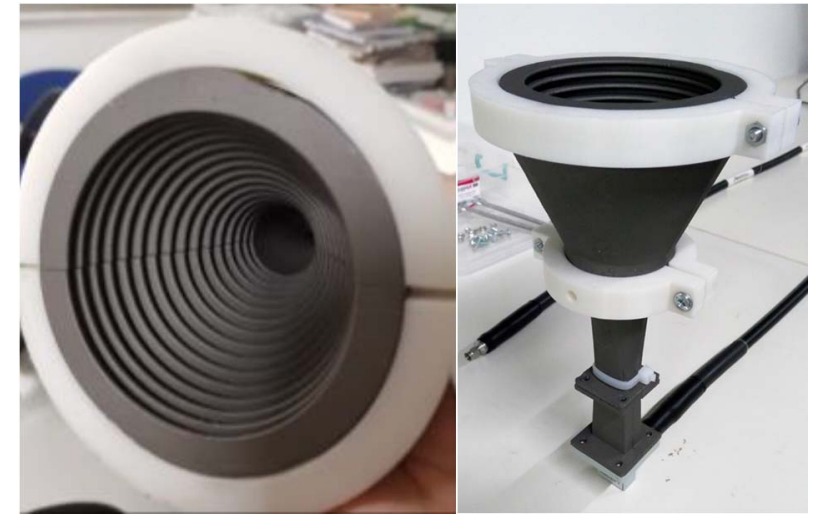

(b)

Figure 6: The manufactured views of the antenna prototype (a) produced and coated half pieces (b) Assembled antenna structure.
Total production time of the antenna is about 23 hours, and total $25.3 \mathrm{~m} / 200 \mathrm{gr}$ of material is used. After printing, the identical parts are coated by using three coats of Super Shield-841 nickel conductive aerosol paint spray to give the coating thickness of approximately $0.1 \mathrm{~mm}$. Finally, the fabricated half-pieces are assembled by combining them with a special adjustable clamp system as shown in Figure 6(b). After the manufacturing steps are completed, the total weight of the prototype antenna is measured as about 230 gr, which is sufficiently lightweight.

\section{Measurement Results and Comparison with Simulation Results}

After the manufacturing steps of the antenna given in Section 3 are completed, the performances (return loss, realized gain, directivity and radiation patterns) of the fabricated antenna are measured. The measurements for return loss and gain performances of the antenna are carried out in Antenna and Microwave Laboratory of Yasar University with a coax-to-WR62 adapter, a standard gain horn antenna, and a vector network analyzer of Anritsu MS2028C where the setup for $\mathrm{S}_{11}$ (return loss) measurement is depicted in Figure 7(a). The directivity and radiation patterns are measured at Planar Near Field Measurement System of Antenna Test and Research Center in TUBITAK BILGEM as shown in Figure 7(b).

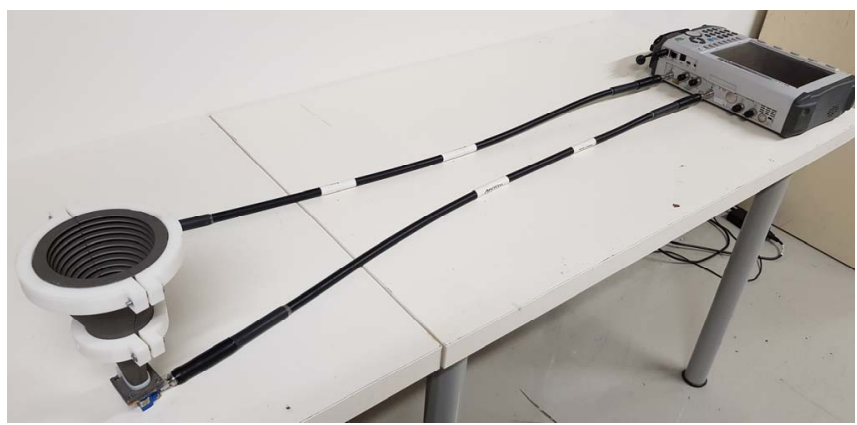

(a)

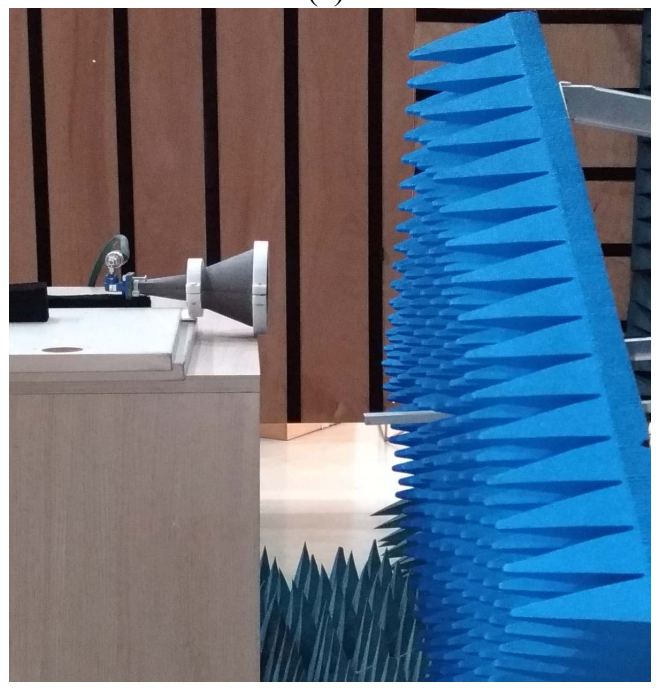

(b)

Figure 7: The photographs of the measurements in (a) Yasar University (b) TUBITAK BILGEM. 
The measurement results of the manufactured antenna for $\mathrm{S}_{11}$ parameter (reflection coefficients) are presented in Figure 8(a) along with the simulation results for the antenna with PLA/nickel coating. As shown from the results in Figure 8(a), the return loss of the antenna is measured to be above $20 \mathrm{~dB}$ at almost whole band of 10.5-18.5 GHz. The reflection coefficients exceed $-20 \mathrm{~dB}$ and reach the value of $-17 \mathrm{~dB}$ at most on the frequencies around $10.5 \mathrm{GHz}$. This is probably due to the reason of non-uniformity in the coating thickness occurred in spray process step of manufacturing. At some parts/points inside the antenna, the thickness may be greater than $0.1 \mathrm{~mm}$, which makes the cutoff frequency approach to $10.5 \mathrm{GHz}$ more and disturbs the reflection response at these frequencies. Besides, the difference between real surface resistance of nickel coating and estimated one used in the simulations can cause the decrease in the return loss performance where nonuniformity in the coating thickness and deviation in surface resistance are also the possible reasons for the slight differences between measured and simulated return loss values at the other frequencies apart from $10.5 \mathrm{GHz}$.

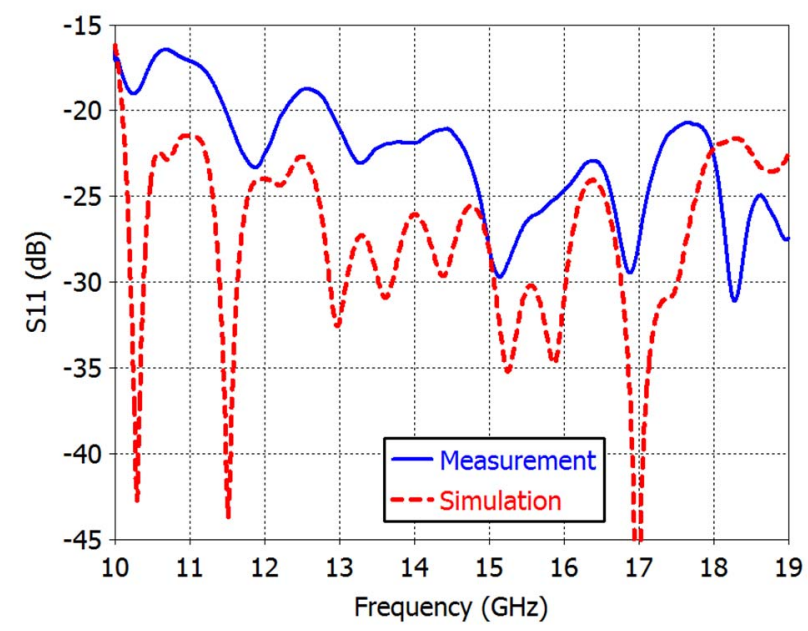

(a)

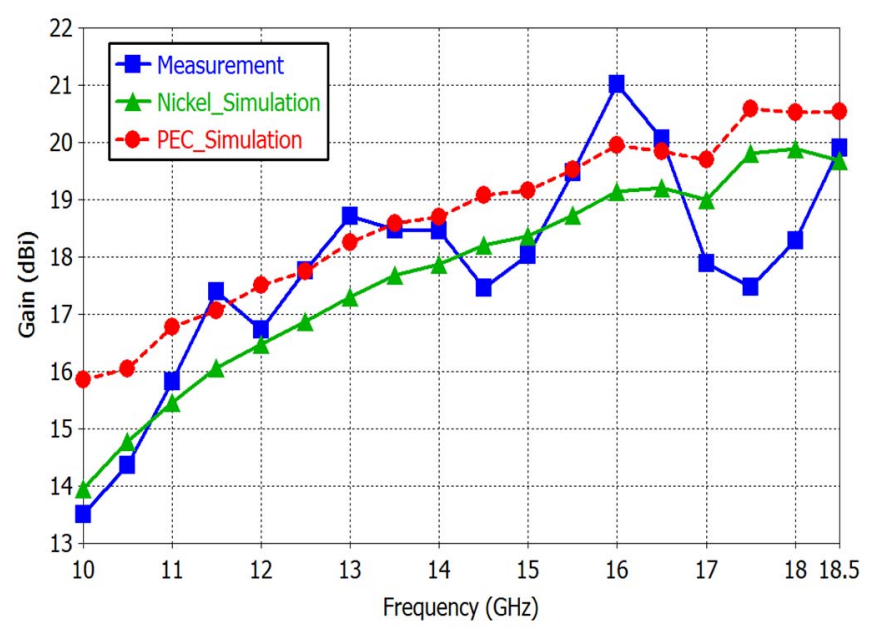

(b)

Figure 8: The simulation and measurement results of the antenna manufactured with 3-D printing and coating technique for (a) $\mathrm{S}_{11}$ parameter and (b) gain.
In spite of small deviations with simulation results, the return loss of the proposed antenna is concluded to be highly adequate with minimum value of $17 \mathrm{~dB}$ in the band of 10.5-18.5 GHz that the values are even higher than $20 \mathrm{~dB}$ in the majority part of the band.

The measured realized gain of the proposed antenna is demonstrated in Figure 8(b) along with the simulated gain values obtained from the structure with PLA/nickel and PEC material. Here, the simulation results belonging to PEC material are added for the purposes of directivity comparison to be given in the following part and evaluating the gain loss of the antenna given in this study as compared to the antenna if manufactured with $\mathrm{CNC}$ machine/aluminum technique. As it can be observed from the gain results of simulation (with 3-D printing/nickel coating) and measurement, the values are in good agreement that slight differences of approximately $2 \mathrm{~dB}$ at most are again caused by the reasons described in the preceding paragraphs as well as the possible errors in the manufacturing steps related with 3-D printing. Furthermore, when the measured gain values are compared with those of PEC material, it is achieved a gain loss of about $2 \mathrm{~dB}$ with respect to the same antenna structure fabricated with $\mathrm{CNC}$ machine. However, even with the mentioned moderate gain loss, the manufactured antenna with the proposed technique is measured to give minimum gain value of $14.5 \mathrm{dBi}$ for the frequency band of $10.5-18.5 \mathrm{GHz}$ where the peak gain value reaches almost $21 \mathrm{dBi}$ within the band.

In the measurement of radiation patterns, since the facility of TUBITAK BILGEM Near Field Measurement System can provide radiation patterns up to $18 \mathrm{GHz}$, the radiation pattern measurements are realized at $10.5 \mathrm{GHz}, 14.5 \mathrm{GHz}$, and $18 \mathrm{GHz}$. The corresponding radiation patterns at the mentioned frequencies are given in Figure 9 and Figure 10 for E-plane (elevation) and H-plane (azimuth), respectively. Here, the normalized co-polarization patterns are given for both measurement and simulation with PLA/nickel for the comparison. It can be observed from the results given in Figure 9 and Figure 10 that the simulation and measurement results are sufficiently close to each other by giving peak sidelobe level of $-18 \mathrm{~dB}$ at most (for the case of $10.5 \mathrm{GHz}$ in E-plane) in measurement.

As the final analysis in this section, the directivity values acquired from the simulations and measurement results are compared. The directivity values of simulation are taken from the gain values of PEC material given in Section 2.1. The measured directivity values are calculated from the measured half power beamwidth values in E-plane and $\mathrm{H}$ plane and the equations given by

$$
\begin{gathered}
D_{[1]}(d B)=10 \log \left(\frac{26000}{\theta_{E}^{\circ} \theta_{H}^{\circ}}\right) \\
D_{[20]}(d B)=10 \log \left(\frac{72815}{\left(\theta_{E}^{\circ}\right)^{2}+\left(\theta_{H}^{\circ}\right)^{2}}\right)
\end{gathered}
$$

where $\theta_{E}^{\circ}$ and $\theta_{H}^{\circ}$ are half power $(3-\mathrm{dB})$ beamwidth in degrees for E-plane and H-plane, respectively. 


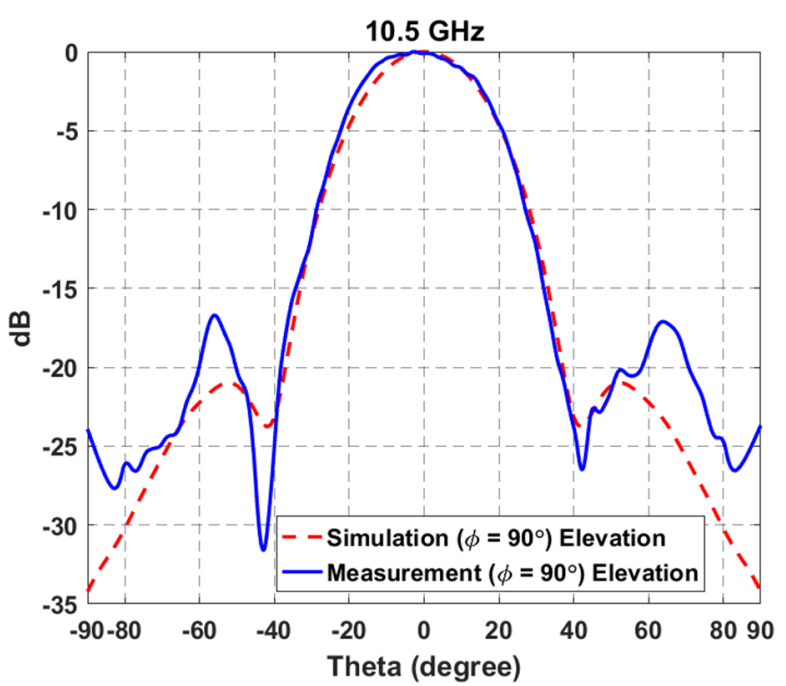

(a)

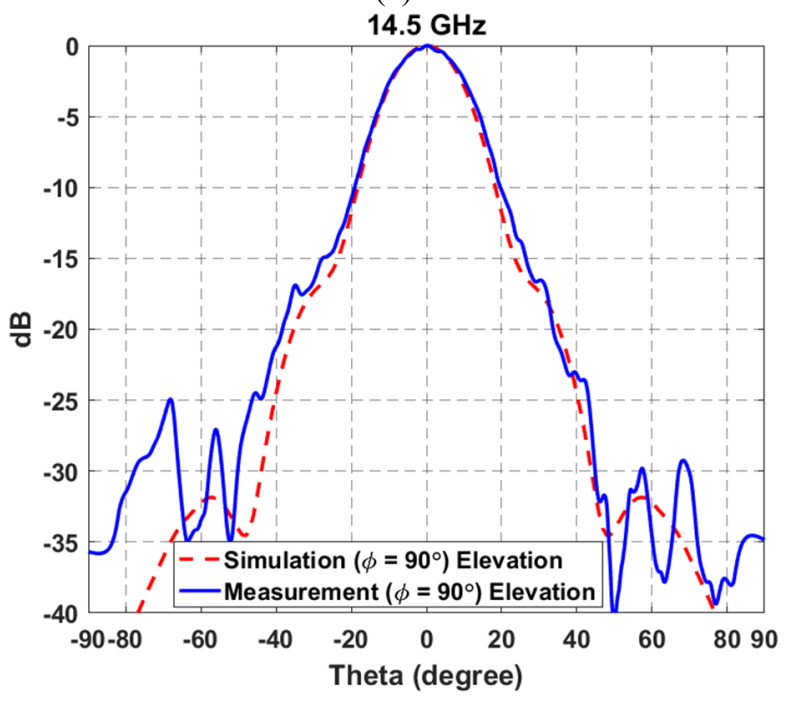

(b)

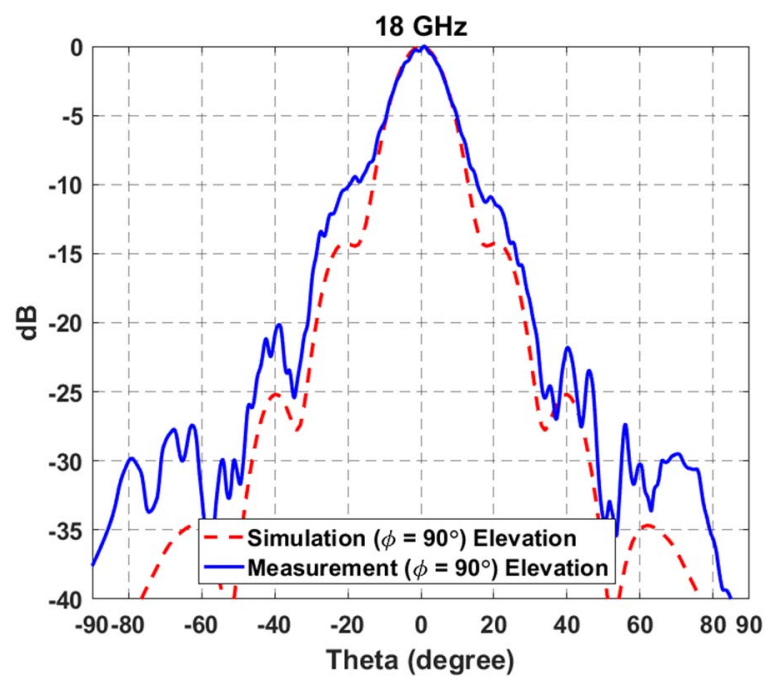

(c)

Figure 9: The measured and simulated (with PLA/nickel) co-polarization normalized patterns for E-plane (elevation) at (a) $10.5 \mathrm{GHz}$ (b) $14.5 \mathrm{GHz}$ (c) $18 \mathrm{GHz}$.

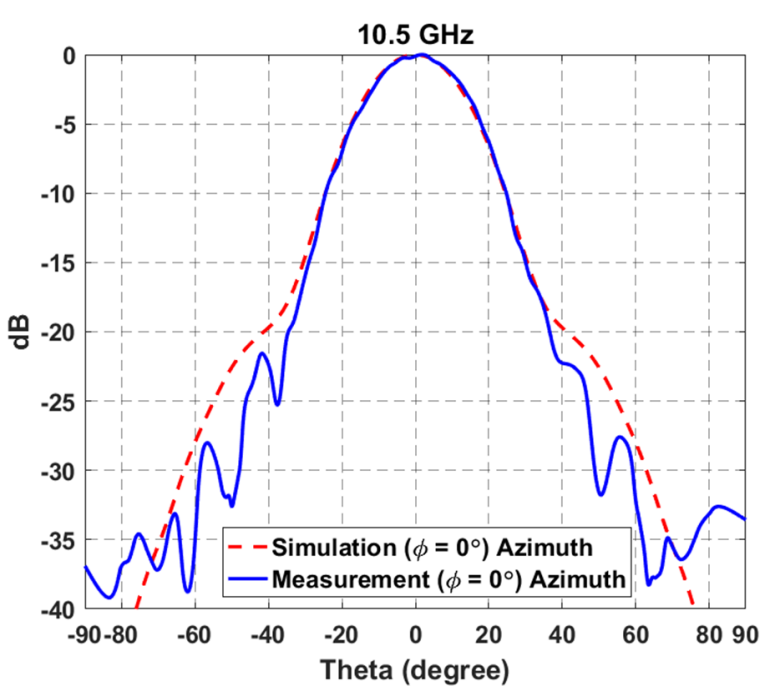

(a)

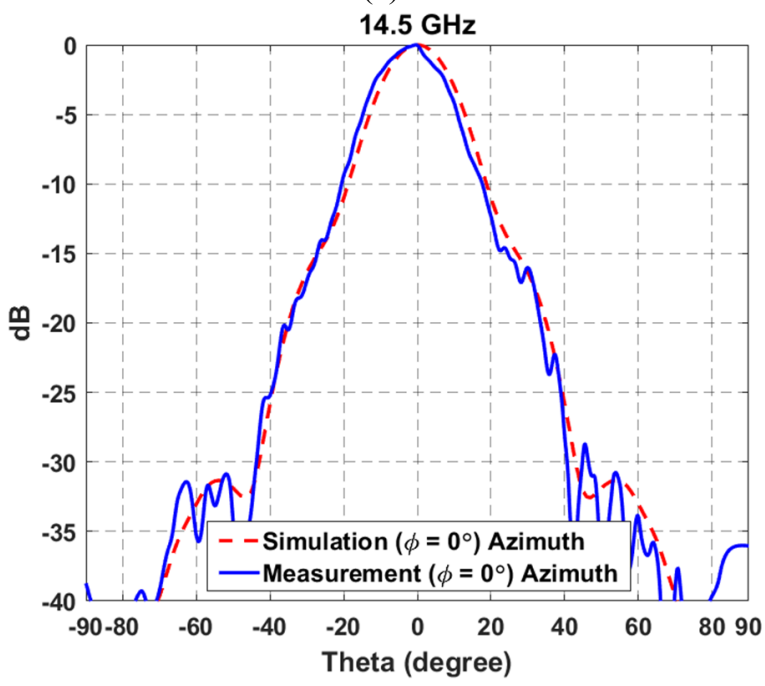

(b)

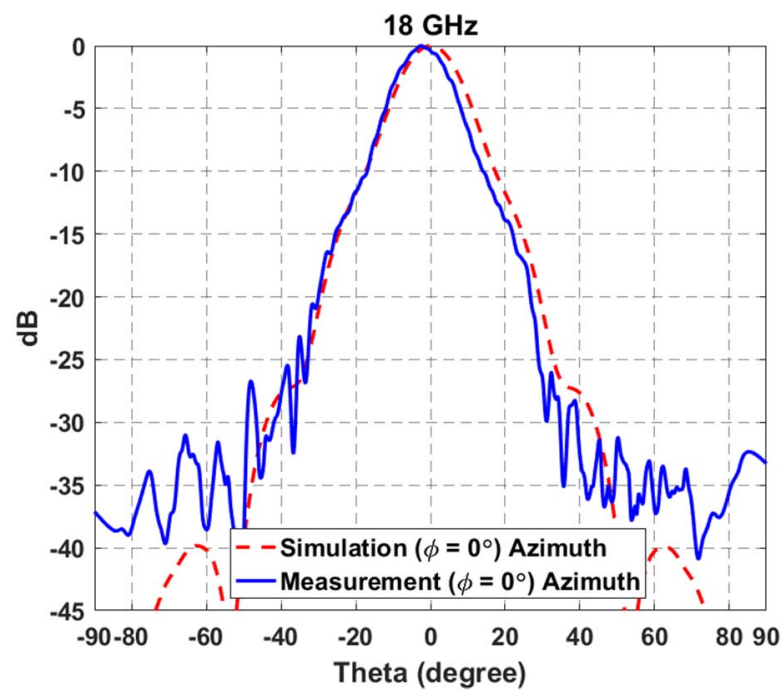

(c)

Figure 10: The measured and simulated (with PLA/nickel) co-polarization normalized patterns for H-plane (azimuth) at (a) $10.5 \mathrm{GHz}$ (b) $14.5 \mathrm{GHz}$ (c) $18 \mathrm{GHz}$. 
Table 2: The comparison of the directivity values obtained from simulations and measurement/equations.

\begin{tabular}{cccc}
\hline $\begin{array}{c}\text { Frequency } \\
{[\mathrm{GHz}]}\end{array}$ & $\begin{array}{c}\text { Simulation } \\
{[\mathrm{dB}]}\end{array}$ & $\begin{array}{c}\text { From (4) } \\
{[\mathrm{dB}]}\end{array}$ & $\begin{array}{c}\text { From (5) } \\
{[\mathrm{dB}]}\end{array}$ \\
\hline 10.5 & 16.05 & 14.23 & 15.55 \\
14.5 & 19.07 & 17.71 & 19.16 \\
18 & 20.52 & 20.60 & 21.77 \\
\hline
\end{tabular}

Here, the equation (4) from [1] usually underestimates the directivity value by considering the worst case when there is not enough information about field distribution at the aperture of the horn antenna, while the equation of (5) from [20] gives more accurate results for high directivity values. The directivity values achieved from simulation and measurement are given in Table I such that the measurement results obtained with (5) give more reliable results with simulation by deviating $1 \mathrm{~dB}$ at most in the band. The values for (4) are found to underestimate the directivities more than $1.5 \mathrm{~dB}$ at some frequencies as explained before.

\section{Discussion}

As described in Section 1, there exist studies in literature, which proposes antennas manufactured with similar technique of 3-D printing/coating [4-7]. However, among these studies, although the study [7] contains a conical horn antenna, its bandwidth even for Return Loss (RL) $>10 \mathrm{~dB}$ is too narrow with a frequency band of 7.4-7.89 GHz (1.066:1 bandwidth). The study [6] demonstrates about a ridge horn antenna in Ka-band manufactured with 3-D printing/stereolithography technique. Although the $10-\mathrm{dB}$ return-loss bandwidth of the antenna is considerably wide from 19 to $31 \mathrm{GHz}$ (1.63:1 bandwidth), it performs quite low frequency bandwidth for $20 \mathrm{~dB}$ return loss or even for $15 \mathrm{~dB}$ return loss. Therefore, the horn antennas in $[4,5]$, which are broadband for high return loss values, i.e. 17 or 20 $\mathrm{dB}$; are used for the comparison where the study [5] makes the manufacturing with relatively expensive and long lead time process of metal plating for coating, and the study [4] uses ABS instead of PLA in the 3-D printing. The initial comparison is done in terms of return loss (RL) frequency bands (bandwidth) and measured peak sidelobe level at the center frequency of the bandwidth where the corresponding results are tabulated in Table 3 .

Table 3: Comparison of return loss frequency band and peak sidelobe level at center frequency.

\begin{tabular}{cccc}
\hline & This study & $\begin{array}{c}\text { Antenna } \\
\text { in Ref } \\
{[4]}\end{array}$ & $\begin{array}{c}\text { Antenna in } \\
\text { Ref. [5] }\end{array}$ \\
\hline $\begin{array}{c}\text { Band (RL } \geq 17 \\
\text { dB) }[\mathrm{GHz}]\end{array}$ & $10.5-19$ & $12.5-18.8$ & $10-15$ \\
$\begin{array}{c}\text { Band (RL } \geq 20 \\
\text { dB) }[\mathrm{GHz}]\end{array}$ & $\begin{array}{c}11.5-12.3 \\
\& 12.9-19\end{array}$ & $13-18.5$ & $10.7-10.8$ \\
$\begin{array}{c}\text { Peak Sidelobe } \\
\text { at Center Freq. } \\
\text { [dB] }\end{array}$ & -25 & -20 & -15 \\
\hline
\end{tabular}

When the bandwidth performances for return loss of the antennas are examined, the proposed antenna in this study gives widest bandwidth for both $17-\mathrm{dB}$ and $20-\mathrm{dB}$ return losses. Besides, the peak sidelobe level performance of the demonstrated antenna is superior to other antennas by giving lower sidelobe levels. The measured gain values of our study and the antennas in [4] and [5] are also compared in Table 4. According to gain values, although the gain values up to $11 \mathrm{GHz}$ is higher in [5], the antenna in this study gives better gain values at above frequencies. The gain values of this study and [5] are similar for upper frequencies greater than $14 \mathrm{GHz}$; but, the proposed antenna significantly supplies higher gain values in the lower frequencies.

Table 4: Comparison of measured gain values.

\begin{tabular}{cccc}
\hline $\begin{array}{c}\text { Frequency } \\
{[\mathrm{GHz}]}\end{array}$ & $\begin{array}{c}\text { This } \\
\text { study } \\
{[\mathrm{dBi}]}\end{array}$ & $\begin{array}{c}\text { Antenna } \\
\text { Ref. [4] } \\
{[\mathrm{dBi}]}\end{array}$ & $\begin{array}{c}\text { Antenna } \\
\text { Ref. [5] } \\
{[\mathrm{dBi}]}\end{array}$ \\
\hline 10 & 13.5 & 6.23 & 15.5 \\
11 & 15.8 & N/A & 15.8 \\
12 & 17 & 15.54 & 16.75 \\
14 & 18.5 & 18.66 & 17.5 \\
16 & 21 & 19.61 & N/A \\
18 & 18.5 & 18.98 & N/A \\
\hline
\end{tabular}

As the final discussion, the manufacturing techniques of 3-D printing/PLA/nickel and CNC-based milling/aluminum are compared in terms of weight, production time and cost. The comparison is shown in Table 5 that the proposed technique presents much lighter antenna and provides cheaper manufacturing at shorter time.

Table 5: Comparison of manufacturing techniques.

\begin{tabular}{lcc}
\hline & $\begin{array}{c}\text { Proposed } \\
\text { (3-D/PLA } \\
\text { and Nickel) }\end{array}$ & $\begin{array}{c}\text { CNC-based } \\
\text { milling and } \\
\text { Aluminum }\end{array}$ \\
\hline Weight [gr] & $232^{(1)}$ & $423^{(2)}$ \\
Production Time [days] & 1.5 & $10-12$ \\
Cost [\$] & $\sim 200$ & $2500-3000$ \\
\hline (1) Including the clamp system \\
(2) The weight of only antenna from CST when pure aluminum with \\
density of $\rho=2700 \mathrm{~kg} / \mathrm{m}^{3}$ is used
\end{tabular}

\section{Conclusions}

In this study, it is aimed to realize a prototype of a conical corrugated horn antenna to be used in $\mathrm{Ku}$-Band satellite communication systems with a low cost and short lead time manufacturing method. For this purpose, technique of 3D printing technology with PLA and metal spray coating is used to produce an antenna operating at wide frequency band between 10.5 and $18.5 \mathrm{GHz}$. Although a conductive material (nickel) with moderate conductivity is used for spray coating with a thickness of about $0.1 \mathrm{~mm}$, it is found from the measurements that the antenna gives not more than $2 \mathrm{~dB}$ gain loss as compared to same antenna manufactured with high conductivity material and CNC-based manufacturing. The proposed antenna structure manufactured with proposed technique still has highly 
satisfactory performances such that it provides more than 17 $\mathrm{dB}$ return loss, $14.5 \mathrm{dBi}$ gain and peak sidelobe level of -18 $\mathrm{dB}$ in E-plane and H-plane at the given frequency band (1.76:1 bandwidth). The proposed fabrication technique has the advantages of low weight, low cost and low production time as compared to CNC-based production in spite of slight loss in the gain. As a conclusion, 3-D printing and coating method is very useful especially for research and prototype verification in antenna and microwave systems.

\section{Acknowledgements}

We would like to give special thanks to Goksenin BOZDAG from TUBITAK BILGEM Antenna Test and Research Center for his helps during the measurements of antenna radiation patterns in this study.

\section{References}

[1] W.L. Stutzman and G.A. Thiele, Antenna Theory and Design, $3^{\text {rd }}$ edition, New Jersey, USA: Wiley, 2013.

[2] R. Noorani, 3D Printing Technology, Applications and Selection, New York, NY, USA: Taylor \& Francis Group, 2018.

[3] J. Uher, J. Bornemann, and U. Rosenberg, Waveguide Components for Antenna Feed Systems: Theory and $C A D$, Boston, USA: Artech House, 1993.

[4] J. C. S. Chieh, B. Dick, S. Loui, and J. D. Rockway, "Development of a Ku-band corrugated conical horn using 3-D print technology," IEEE Antennas and Wireless Propag. Lett., vol. 13, pp. 201-204, 2014. doi:10.1109/LAWP.2014.2301169

[5] A. Genc, I. B. Basyigit, T. Goksu and S. Helhel, "Investigation of the performances of $\mathrm{X}-\mathrm{Ku}$ band 3D printing pyramidal horn antennas coated with the different metals," in 2017 10th International Conference on Electrical and Electronics Engineering (ELECO), Bursa, Turkey, 2017, pp. 1012-1016.

[6] J. S. Silva, M. García-Vigueras, T. Debogović, J. R. Costa, C. A. Fernandes and J. R. Mosig, "Stereolithography-based antennas for satellite communications in Ka-band," Proceedings of the IEEE, vol. 105, no. 4, pp. 655-667, 2017. doi:10.1109/JPROC.2016.2633898.

[7] A. T. Castro, B. Babakhani, and S. K. Sharma, "Design and development of a multimode waveguide corrugated horn antenna using 3D printing technology and its comparison with aluminium-based prototype," IET Microwaves, Antennas \& Propagation, vol. 11, no. 14, pp. 1977-1984, 2017. doi:10.1049/iet-map.2016.0808.

[8] E. Menargues et al., "3D printed feed-chain and antenna components," in 2017 IEEE International Symposium on Antennas and Propagation \& USNC/URSI National Radio Science Meeting, San Diego, CA, 2017, pp. 1-2.

[9] E. Laplanche et al., "A Ku-band diplexer based on $3 \mathrm{~dB}$ directional couplers made by plastic additive manufacturing," in 2017 47th European Microwave Conference (EuMC), Nuremberg, 2017, pp. 428-431.
[10] A. I. Dimitriadis et al., "Polymer-based additive manufacturing of high-performance waveguide and antenna components," Proc of the IEEE, vol. 105, no. 4, pp. 668-676, 2017. doi:10.1109/JPROC.2016.2629511.

[11]X Shang et al., "W-Band Waveguide Filters Fabricated by Laser Micromachining and 3-D Printing," IEEE Trans. Microw. Theory Techn., vol. 64, no. 8, pp. 25722580, 2016. doi:10.1109/TMTT.2016.2574839.

[12]E. Menargues et al., "Polymer-based metal coated additive manufactured $\mathrm{V}$ - and $\mathrm{W}$-band antenna feed chain components," 2017 11th European Conference on Antennas and Propagation (EUCAP), Paris, France, 2017, pp. 584-588.

[13]B. R. Elbert, Satellite Communication Applications Handbook, $2^{\text {nd }}$ edition, Boston, USA: Artech House, 2004.

[14]C. Turkmen, and M. Secmen, "Omnidirectional and circularly polarized slotted antenna array with increased bandwidth performance by using non-Identical waveguide slots", Radio Science RS006635, 2018. doi:10.1029/2018RS006635

[15] C. Granet and G. L. James, "Design of corrugated horns: A primer," IEEE Antennas and Propagation Magazine, vol. 47, no. 2, pp. 76-84, 2005. doi:10.1109/MAP.2005.1487785.

[16]M.F. Yakan Musthofa and A. Munir, "Design of rectangular to circular waveguide converter for S-band frequency," in Proc. 2011 International Conference on Electrical Engineering and Informatics, Bandung, 2011, pp. 1-5.

[17] MG Chemicals, Surrey, BC, Canada, "Super Shield nickel conductive coating 841 technical data sheet," [Online]. Available: http://www.mgchemicals.com

[18]MG Chemicals, Surrey, BC, Canada, "Super Shield silver coated copper conductive coating 843 technical data sheet," [Online]. Available: http://www.mgchemicals.com

[19] S.Rao, S.K. Sharma, and L.Shafai, Handbook of Reflector Antennas and Feed Systems, vol. 2, Boston, USA: Artech House, 2013.

[20]C. A. Balanis, Antenna Theory Analysis and Design, $4^{\text {th }}$ edition, New Jersey, USA: Wiley, 2016. 